\title{
Avaliação do volume representativo elementar de uma rocha carbonática heterogênea utilizando-se microtomografia computadorizada de raios $\mathrm{X}$ e simulação numérica
}

William Godoy* (COPPE/UFRJ), Thaís Silveira (COPPE/UFRJ), Paulo Couto (EP/UFRJ), José Alves (COPPE/UFRJ), Edmilson Rios (COPPETEC/UFRJ), Leonardo Borghi (IGEO/UFRJ)

Copyright 2016, SBGf - Sociedade Brasileira de Geofísica

Este texto foi preparado para a apresentação no VII Simpósio Brasileiro de Geofísica, Ouro Preto, 25 a 27 de outubro de 2016. Seu conteúdo foi revisado pelo Comitê Técnico do VII SimBGf, mas não necessariamente representa a opinião da SBGf ou de seus associados. É proibida a reprodução total ou parcial deste material para propósitos comerciais sem prévia autorização da SBGf.

\section{Abstract}

Carbonate rocks present several challenges regarding the analysis of petrophysical parameters and full understanding of the dynamics of fluid flow in their porous systems. Techniques using X-Ray microtomography, three-dimensional digital model reconstruction of these rocks, and the simulation of fluid flow in the reconstructed porous systems have been continuously developed and studied. This paper presents a possible approach to the analysis of the Representative Elementary Volume (REV) according to the estimates of porosity and absolute permeability in a stromatolite sample using Digital Rock Physics, involving all steps necessary for the reconstruction of porous systems and numerical simulation of fluid flow in different sample subvolumes in a region of interest $(\mathrm{ROI})$.

\section{Introdução}

A microtomografia computadorizada de raios $X$ (microCT, MCT ou $\mu C T$ ), também denominada de tomografia computadorizada de alta resolução, pode ser definida como uma técnica não destrutiva que permite a obtenção e análise de centenas de seções ou imagens microtomográficas e possibilita a posterior reconstrução e visualização tridimensional das regiões internas das amostras analisadas, além de quantificações automatizadas de área e/ou volume (Neto et al., 2011).

Muitos trabalhos abordaram a utilização da técnica de microtomografia para o estudo de carbonatos e suas respectivas propriedades petrofísicas, com destaque para Kaczmarczyk et al. (2010) e Kaczmarczyk et al. (2011), que abordaram a comparação dos valores de porosidade e permeabilidade absoluta obtidos através de dados provenientes de micro-CT e de dados experimentais, Youssef et al. (2007), que validaram os modelos porosos 3D criados a partir de dados de micro-CT em função das estimativas de porosidade e permeabilidade. Estes estudos, entretanto, não se propuseram a analisar o Volume Representativo Elementar em função dos dados obtidos.

O Volume Elementar Representativo ("Representative Elementary Volume" - REV) pode ser definido como o volume de uma amostra capaz de capturar uma quantidade representativa de sua heterogeneidade (Bear, 1972). Assim, o REV pode ser considerado como o volume no qual as propriedades macroscópicas (como a condutividade hidráulica ou a permeabilidade absoluta, por exemplo) são relativamente insensíveis a pequenas mudanças de volume da amostra analisada ou da localização da região de interesse dentro desta mesma amostra (Corbett, 2009). O REV pode diferir em dimensão de acordo com a propriedade petrofísica que se deseje mensurar (Vik et al., 2013a), de tal forma que uma escala representativa adequada pode depender diretamente da propriedade avaliada momentaneamente. Com o REV, flutuações nas medidas das propriedades estudadas são inexistentes e uma quantidade representativa de heterogeneidade pode ser estabelecida, conforme apresentado na figura 1.

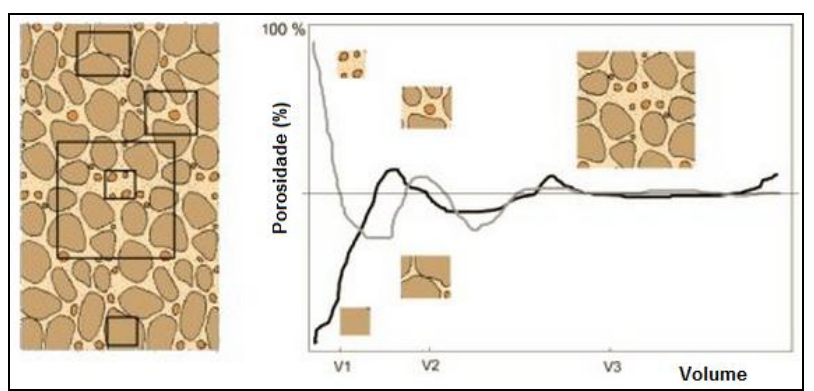

Figura 1 - Caracterização do REV a partir das medidas de porosidade em uma amostra. Fonte: Modificado de BEAR (1972).

O presente trabalho realiza um estudo acerca da análise do REV em função de dois diferentes parâmetros petrofísicos: a porosidade e a permeabilidade absoluta. Ambos são estimados a partir de uma amostra de estromatólito submetida à técnica de microtomografia computadorizada de raios $X$ e posteriormente à técnica de petrofísica digital, incluindo-se a simulação numérica do escoamento de um fluido no sistema poroso digitalmente reconstruído.

Estudou-se o escoamento monofásico de fluidos em nível de poros, e três trabalhos (Fourie et al., 2007; Narsilio et al., 2009; Kaczmarczyk et al., 2011) foram utilizados como referência, pois estudaram a possibilidade de se fazer um upscaling das equações de Navier-Stokes, que regem 0 escoamento de fluidos na microescala, utilizando-as para se obter parâmetros associados ao escoamento na mesoescala, de forma a se obter estimativas de parâmetros, como a permeabilidade absoluta, por exemplo. Estas estimativas de permeabilidade absoluta foram utilizadas, então, para a análise do REV em função dos volumes amostrais obtidos da região de interesse da amostra e comparadas com o REV obtido a partir das estimativas de porosidade. 


\section{Método}

A amostra carbonática foco deste estudo é oriunda das imediações da Lagoa Salgada, localizada no estado do Rio de Janeiro, tendo sido coletada a partir de construções estromatolíticas superficiais presentes nas margens da lagoa. Esta mesma amostra foi alvo de estudos conduzidos por Hayashi (2014).

A etapa de aquisição de dados a partir da técnica de microtomografia computadorizada de raios $\mathrm{X}$ foi realizada utilizando-se o microtomógrafo modelo GE phoenix $v$ /tome $/ x \mathrm{~m}$. Os parâmetros da microtomografia podem ser observados na tabela 1.

Tabela 1- Parâmetros relativos à aquisição de dados por microtomografia de raios $\mathrm{X}$ da da amostra estudada.

\begin{tabular}{|l|l|}
\hline \multicolumn{2}{|l|}{ Geometria } \\
\hline Tamanho do Voxel & $106,55 \mu \mathrm{m}$ \\
\hline Detector & $\mathrm{dxr}-250$ \\
\hline Tipo & $150 \mathrm{kV}$ \\
\hline Raios X & $700 \mu \mathrm{A}$ \\
\hline Voltagem & $0,1 \mathrm{~mm} \mathrm{Cu}$ \\
\hline Corrente & \\
\hline Filtro &
\end{tabular}

A partir da análise de suas distintas estruturas morfológicas internas, pôde-se identificar três diferentes estágios de crescimento do estromatólito. Selecionou-se a região basal do estromatólito, denominada Base ou Cellar com as seguintes dimensões aproximadas: $6,4 \mathrm{~cm}$ de comprimento, $5,8 \mathrm{~cm}$ de largura e $1,0 \mathrm{~cm}$ de altura, conforme apresentado na figura 2. A partir desta, denominada Cellar 1, escolheu-se uma região de interesse (ROI), deñominada Cellar_2, com dimensões de $3,2 \mathrm{~cm}$ de comprimento, $2,9 \mathrm{~cm}$ de largura e $1,0 \mathrm{~cm}$ de altura, correspondendo, portanto, a um volume de aproximadamente $9,3 \mathrm{~cm}^{3}$.

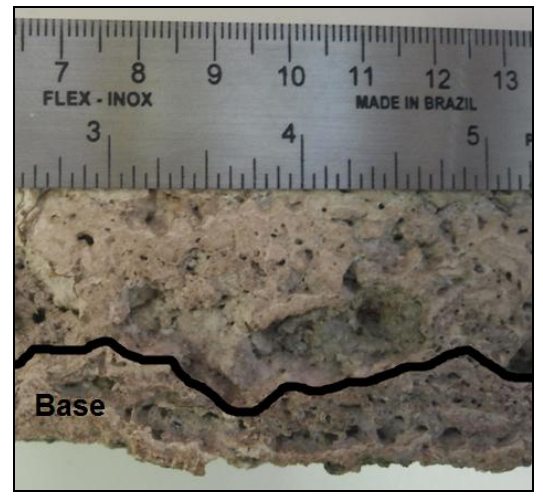

Figura 2 - Amostra de estromatólito utilizada no estudo. A ROI foi selecionada a partir da Base da amostra.

Foram utilizados programas computacionais gratuitos e comerciais (Fiji/lmageJ e Simpleware ScanIP 6.0, respectivamente) para a avaliação das imagens obtidas a partir da microtomografia de raios $\mathrm{X}$, para a reconstrução digital da amostra e de seu sistema poroso.

Uma etapa de extrema importância foi a de segmentação das imagens e definição do valor de limiar (threshold). A segmentação pode ser entendida como um processo de conversão de uma imagem em escala de cinza ou mesmo colorida para uma imagem binária, ou binarizada, através da identificação de duas populações presentes na imagem, baseada na intensidade de seus valores obtidos durante a aquisição tomográfica (Al-Raoush \& Willson, 2005).

Neste estudo foram utilizados três metodologias para a definição do valor de limiar: Método de Otsu (Otsu, 1979), Método baseado na forma do histograma (Kaczmarczyk et al., 2010) e Método dos Contornos ativos sem bordas (Chan \& Vese, 2001), os quais convergiram para o mesmo patamar de valor. A etapa de obtenção do valor de limiar foi realizada com auxílio de programas computacionais gratuitos e comerciais (Fityk 0.9.8 e Matlab 2012b, repectivamente) e algoritmos computacionais.

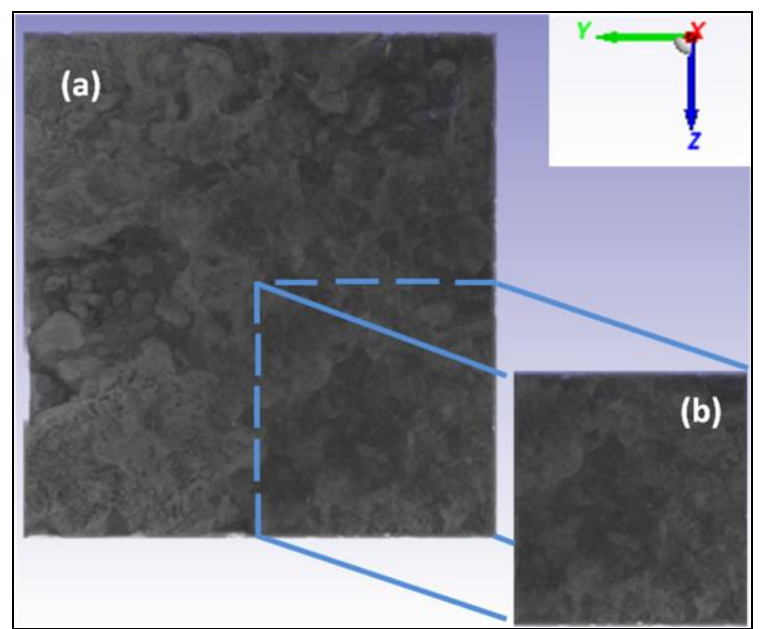

Figura 3 - Representação das regiões (a) Cellar_1 e (b) Cellar 2 selecionadas na camada Base (ou Cellar) a partir de um volume amostral com maiores dimensões.

A partir da definição do valor de limiar e da posterior reconstrução do sistema poroso efetivamente conectado, a região de interesse (ROI) foi subdividida, conforme divisão sugerida na figura 4 . Os subvolumes obtidos pelas divisões de cada uma destas regiões foram denominados da seguinte forma:

1. Para a região denominada Cellar 2, suas duas metades foram chamadas de Cellar 21 e Cellar_2_2, com dimensões aproximadas de: 1,6 $\mathrm{cm}$ de comprimento, $2,8 \mathrm{~cm}$ de largura e $1,0 \mathrm{~cm}$ de altura, cujo volume aproximado é de $4,6 \mathrm{~cm}^{3}$.

2. Cada uma destas duas metades foi novamente dividida, e seus subvolumes foram denominados Cellar_2_1_1 e Cellar_2_1_2, para o volume 
chamado de Cellar_2_1, e Cellar_2_2_1 e Cellar_2_2_2, para ${ }^{-}$volume denominado Cellar_2_2. As dimensões aproximadas para cada um destes subvolumes são: $1,6 \mathrm{~cm}$ de comprimento, $1,4 \mathrm{~cm}$ de largura e $1,0 \mathrm{~cm}$ de altura, cujo volume aproximado é de $2,3 \mathrm{~cm}^{3}$;

3. Essa forma de nomenclatura sequencial, conforme apresentada no item (2) foi utilizada também para os oito subvolumes de $1,15 \mathrm{~cm}^{3}$, dezesseis subvolumes de $0,575 \mathrm{~cm}^{3}$ e os trinta e dois subvolumes subsequentes de $0,288 \mathrm{~cm}^{3}$ analisados. Estes subvolumes menores não estão esquematizados na figura 4 , mas seguem a mesma lógica proposta.

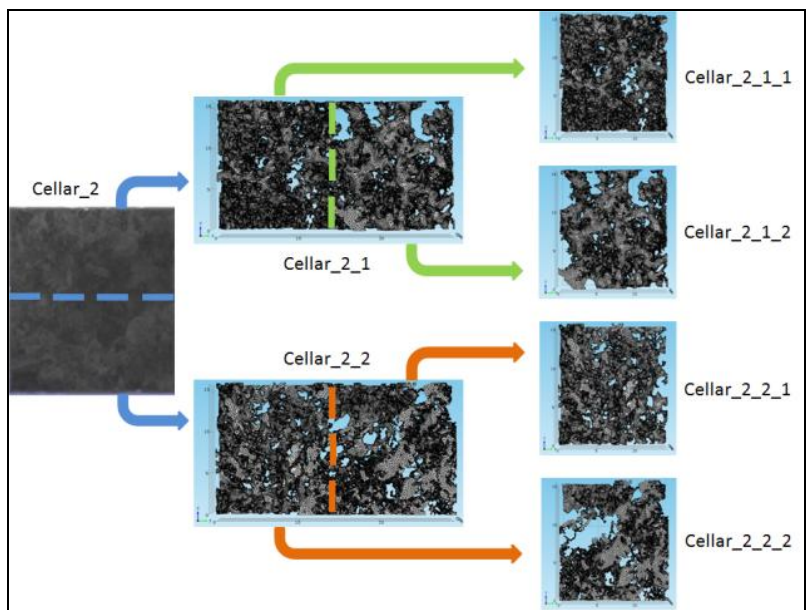

Figura 4 - Esquema de divisão para obtenção dos subvolumes a partir da região denominada Cellar_2.

Cada um desses subvolumes teve seu sistema poroso modelado. Foram geradas malhas representativas da geometria modelada através de um programa computacional comercial (Simpleware +FE 6.0). A porosidade pode ser estimada através da relação entre 0 volume do sistema poroso modelado e o volume total de cada subvolume de interesse.

A estimativa de permeabilidade absoluta para cada subvolume foi obtida através da modelagem do sistema poroso efetivamente conectado, novamente a partir da malha representativa da geometria de interesse. A simulação numérica foi realizada através de um programa computacional comercial (Comsol Multiphysics 4.4) baseado no Método dos Elementos Finitos (MEF). A formulação matemática para 0 cálculo das permeabilidades absolutas é encontrada nos trabalhos de Narsilio et al. (2009) e Kaczmarczyk et al. (2011), assumindo-se algumas premissas para facilitar a resolução do problema: todas as tensões no campo de escoamento são relacionadas ao meio poroso, o fluido contido na rede de poros possui temperatura e densidade constantes (simulou-se o escoamento monofásico com a água como fluido presente), escoamento laminar (escoamento de Stokes ou Creeping Flow) e foi realizada uma análise estacionária. É importante ressaltar que o custo computacional para a realização das simulações numéricas é diretamente dependente do número de graus de liberdade da geometria de interesse e dos componentes que constituem o computador.

Os graus de liberdade correspondem às incógnitas do problema a ser resolvido (pressão e velocidade, neste caso), que são utilizadas para a montagem de um sistema de equações de grande dimensão, diretamente baseado no modelo obtido. Assim, quanto maior for a quantidade de elementos usados, maior será o sistema de equações e maior será o custo computacional necessário para resolvê-lo. Quanto aos componentes físicos do computador, para a resolução das equações associadas ao problema é necessária uma grande quantidade de RAM e um grande poder de processamento. Neste estudo utilizou-se um computador com 64GB de RAM.

Para a obtenção do perfil de velocidade através de simulação numérica, conforme exemplo apresentado na figura 5 , foi considerado, como condição essencial, um diferencial de pressão extremamente baixo, de forma a se obter um escoamento condizente com as premissas anteriormente estabelecidas.

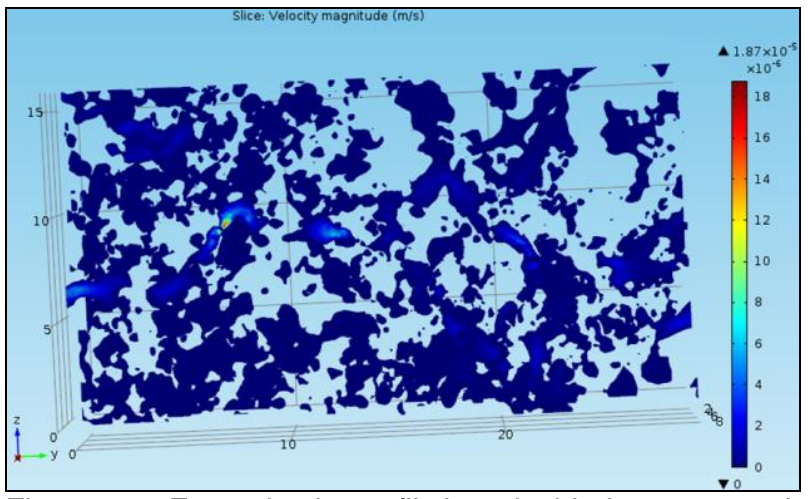

Figura 5 - Exemplo de perfil de velocidade, encontrado através de simulação numérica do escoamento de fluido no sistema poroso efetivamente conectado, de um subvolume da amostra de estromatólito estudada, considerando-se um diferencial de pressão muito baixo.

\section{Resultados}

O gráfico da figura 6 apresenta as estimativas de porosidade total para a região Cellar_2, relacionadas aos volumes amostrais obtidos pelos critérios estabelecidos anteriormente. Estes servirão como comparativos para os gráficos que correlacionam as estimativas de permeabilidade absoluta calculadas através da simulação numérica, considerando-se estes mesmos volumes amostrais. É importante destacar que o valor de porosidade da região selecionada encontra-se na convergência das duas distribuições, conforme esperado ao se analisar a tendência da curva apresentada na 
figura 1. Os valores estão coerentes com as estimativas encontradas no trabalho de Hayashi (2014), cujas estimativas de porosidade foram realizadas na mesma amostra alvo deste estudo.

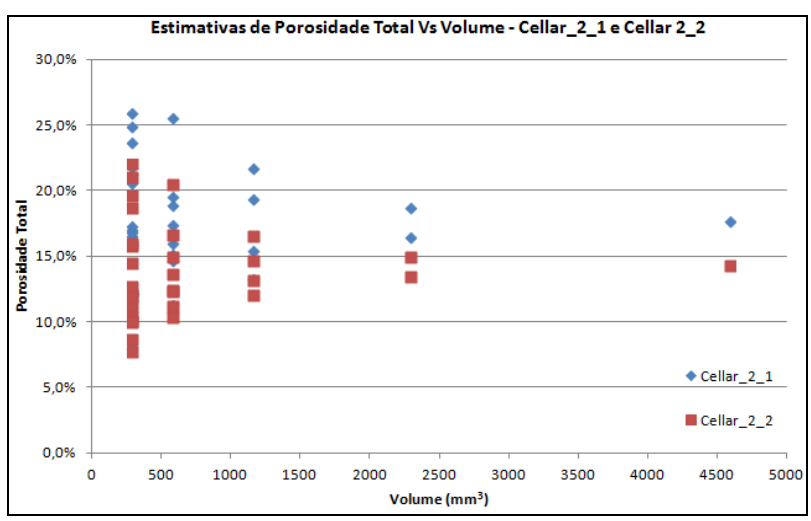

Figura 6 - Correlação entre os volumes e seus respectivos valores de porosidade total obtidos a partir dos dois subvolumes oriundos da divisão da região Cellar 2 .

Já o gráfico da figura 7 apresenta as estimativas de permeabilidade absoluta para o escoamento na direção $z$, conforme orientação apresentada nos eixos presentes na figura 3 , para os subvolumes constituintes da região Cellar 2, obtidos pelo método citado neste trabalho. A solução numérica para a estimativa da permeabilidade do volume correspondente à região Cellar_2. A figura 7 apresenta os comportamentos das estimativas de permeabilidade para os subvolumes Cellar_2_1 e Cellar_2_2 e, como pode ser observado, há uma clara convergência para um patamar bem definido.

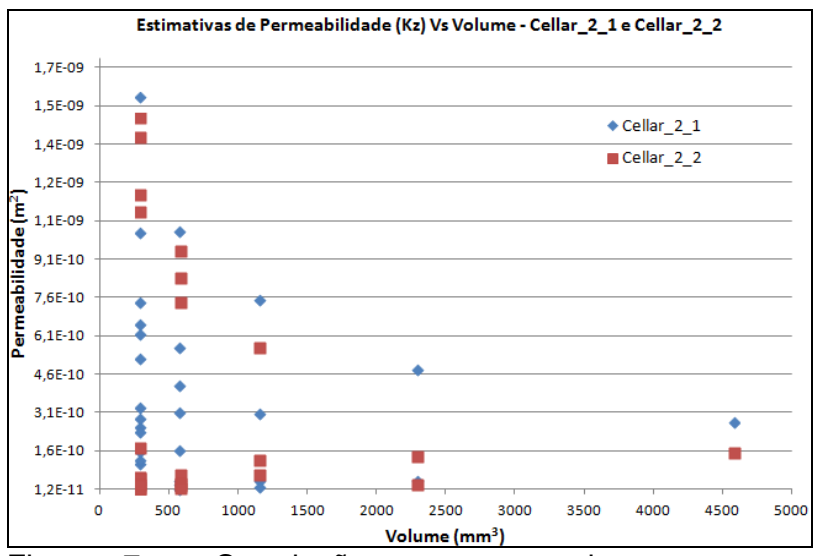

Figura 7 - Correlação entre os volumes e seus respectivos valores de permeabilidade absoluta na direção z obtidos a partir dos dois subvolumes oriundos da divisão da região Cellar 2 .

É importante destacar que todas as simulações numéricas, representadas por cada ponto nos gráficos indicativos das permeabilidades absolutas, foram realizadas para os diversos volumes considerados, e apresentaram convergência e tempos de processamento que variaram entre alguns minutos, para os menores subvolumes, até algumas horas, para os maiores subvolumes utilizando-se um computador com processador core i7 (Intel). Estas convergências se deram tanto para o perfil de velocidades quanto para o de pressões.

Conforme também já mencionado, o REV depende da propriedade que se está medindo. Com os resultados obtidos é possível verificar que, tomando-se um determinado volume amostral e considerando-se a técnica utilizada por Hayashi (2014), no qual o REV é determinado a partir do desvio da média da propriedade referente a todas as medidas realizadas (porosidade ou permeabilidade absoluta, neste caso), tendo sido utilizado em seu trabalho $10 \%$ de desvio máximo em relação à média da porosidade total, a propriedade que se está medindo realmente interfere no REV.

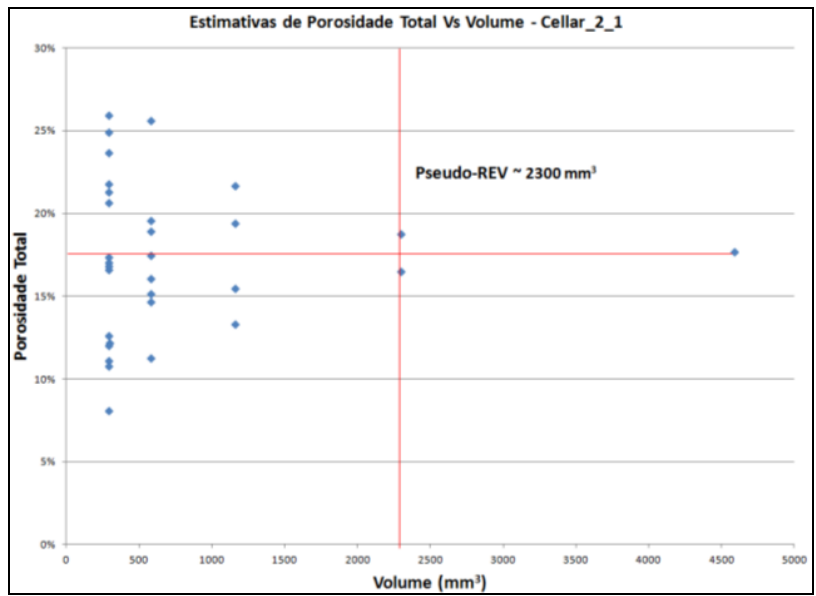

Figura 8 - Gráfico apresentando o pseudo-REV referente à porosidade total da região Cellar_2_1, considerando-se um desvio máximo de $10 \%$ nas estimativas de porosidade total.

Assim, tomando-se a região Cellar 21 e analisando-se tanto os dados referentes à porosidade quanto à permeabilidade absoluta, e definindo-se um pseudo-REV, ou seja, um REV definido para o subvolume da região Cellar_2_1 e, portanto, não passível ser representativo da região Cellar 2, foram obtidos, a partir dos dados estimados, considerando um desvio máximo de $10 \%$ nas estimativas de porosidade total e $20 \%$ nas estimativas de permeabilidade absoluta na direção $z$, os valores de pseudo-REV para cada propriedade petrofísica analisada. Pode-se observar através dos gráficos presentes nas figuras 8 e 9 , uma grande variação no valor do pseudo-REV, sendo o mesmo bem inferior quando se analisa a porosidade total, mesmo utilizandose diferentes valores de desvio médio entre as duas propriedades estimadas. Considerando-se a permeabilidade absoluta como parâmetro petrofísico para a escolha do REV da região de interesse da amostra, 
seria necessário um volume duas vezes maior que aquele necessário para a mesma escolha em função da porosidade total.

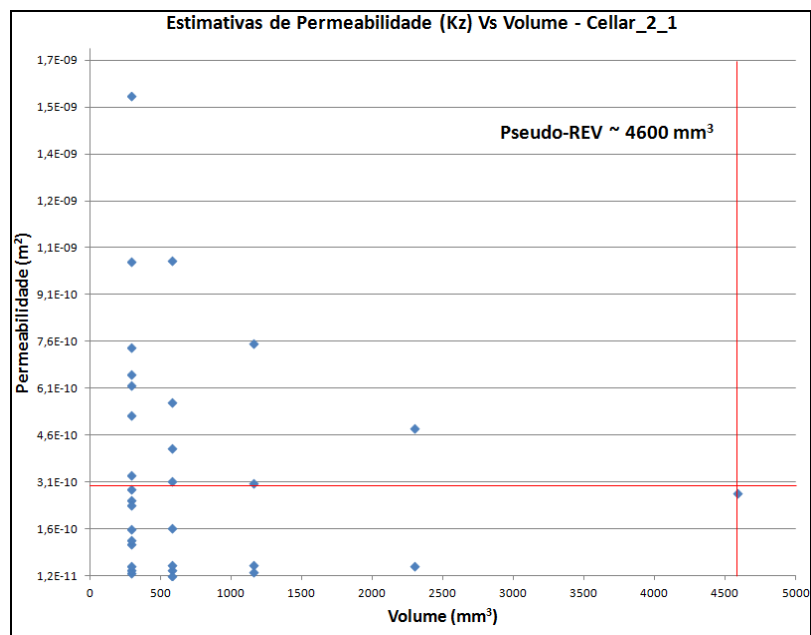

Figura 9 - Gráfico apresentando o pseudo-REV referente à permeabilidade absoluta na direção $\mathrm{z}$ da região Cellar_2_1, considerando-se um desvio máximo de $20 \%$ nas estimativas de permeabilidade absoluta.

\section{Discussão e Conclusões}

O método apresentado neste estudo mostrou-se bastante promissor, podendo ser utilizado para a obtenção das estimativas de porosidade e permeabilidade absoluta e para a avaliação do REV, e num contexto ainda mais amplo, ser inserido em um fluxograma que envolva estudos de técnicas de upscaling. Além disso, os resultados demonstraram que os comportamentos dos parâmetros petrofísicos estimados a partir do estromatólito estudado são coerentes com as observações feitas e com os gráficos construídos para a caracterização do REV em rochas heterogêneas carbonáticas.

Entretanto, um ponto que deve ser destacado refere-se aos altos valores encontrados para as estimativas de permeabilidade absoluta, independentemente do volume amostral utilizado. A compactação afeta as porosidades total e efetiva de uma rocha, e também sua permeabilidade absoluta. A amostra carbonática lacustre estudada neste trabalho está localizada superficialmente, com pouca compactação e, portanto, valores altos para esta propriedade já eram esperados. Entretanto, novos estudos utilizando-se diferentes amostras de subsuperfície devem ser realizados. Para fins de estudo e avaliação do método empregado em rochas carbonáticas heterogêneas, a amostra retirada da Lagoa Salgada foi satisfatória.

Por fim, vale ressaltar que as técnicas de segmentação apresentadas neste trabalho foram selecionadas dentre vários possíveis métodos (Sezgin \& Sankur, 2004), e que a obtenção de um valor de limiar confiável pode ser feita através da utilização de diferentes métodos automatizados, da análise de seus resultados, juntamente com uma caracterização geológica e morfológica apropriada.

\section{Agradecimentos}

Os autores agradecem ao CNPq, à CAPES, à ANP, à BG Brasil / Shell, à Petrobras, ao Laboratório de Instrumentação Nuclear (LIN) da UFRJ / COPPE, ao professor Ricardo Tadeu, aos pesquisadores Alessandra Machado e Átila de Paiva Teles, ao Laboratório de Geologia Sedimentar (LAGESED) da UFRJ / IGEO e ao geólogo Felipe Yuji Hayashi pelo suporte e apoio ao longo da execução do estudo.

\section{Referências}

Al-Raoush, R.I., Willson, C.S., 2005. A pore-scale investigation of a multiphase porous media system. Journal of Contaminant Hydrology 77, 67-89. Elsevier.

Bear, J., 1972. Dynamics of Fluid in Porous Media, Dover Publications inc., pp. 151-173.

Chan, T.F., Vese, L.A., 2001. Active Contours Without Edges. IEEE Transactions on Image Processing, Vol. 10, No. 2.

Corbett, P.W.M., 2009. Integration of static and dynamic models. Petroleum geoengineering. SEG/EAGE Distinguished Instructor Series, vol. 12, pp. 100-190.

Fourie, W., Said, R., Young, P., Barnes, D.L., 2007. The Simulation of Pore Scale Fluid Flow with Real World Geometries Obtained from X-Ray Computed Tomography. COMSOL Conference 2007, Boston, MA, U.S.A.

Hayashi. F.Y.D., 2014. Avaliação do Volume Representativo Elementar (REV) em estromatólito de Lagoa Salgada. Trabalho Final de Curso. Geologia/UFRJ. Rio de Janeiro, RJ - Brasil.

Kaczmarczyk, J., Dohnalik, M., Zalewska, J., 2010. Three-dimensional Pore Scale Fluid Flow Simulation Based on Computed Microtomography Carbonate Rocks' Images. V European Conference on Computational Fluid Dynamic, ECCOMAS CFD 2010.

Kaczmarczyk, J., Dohnalik, M., Zalewska, J., 2011. Evaluation of Carbonate Rock Permeability, with the Use of X-ray Computed Microtomography. Nafta-Gaz Rok LXVII, nr 4.

Narsilio, G., Buzzi, O., Fityus, S., Yun, T.S., Smith, D.W., 2009. Upscaling of Navier-Stokes equations in porous media: Theoretical, numerical and experimental approach. Computers and Geotechnics 36, 7: 12001206. Elsevier. 
Neto, J.M.R., Fiori, A.P., Lopes, A.P., Marchese, C., Pinto-Coelho, C.V., Vasconcellos, E.M.G., Silva, G.F., Secchi, R., 2011. A microtomografia computadorizada de raios $\mathrm{x}$ integrada à petrografia no estudo tridimensional de porosidade em rochas. Revista Brasileira de Geociências 41 (3): 498-508.

Otsu, N., 1979. A Limiar Selection Method from GrayLevel Histograms. IEEE Transactions on Systems, Man, and Cybernetics, SMC-9, 62-66.

Sezgin, M., Sankur, B., 2004. Survey over image limiaring techniques and quantitative performance evaluation. Journal of Electronic Imaging 13(1), 146-165.
Vik, B., Bastesen, E., Skauge, A., 2013. Evaluation of representative elementary volume for a vuggy carbonate rock - Part I: Porosity, permeability, and dispersivity. Journal of Petroleum Science and Engineering, 112, pp. 36-47.

Youssef, S., Rosenberg, E., Gland, N., Kenter, J.A.M., Skalinski, M., Vizika, O., 2007. High Resolution CT and Pore-Network Models to Assess Petrophysical Properties of Homogeneous and Heterogeneous Carbonates.: 2007 SPE/EAGE Reservoir Characterization and Simulation Conference, Abu Dhabi, UAE. 